\title{
Increasing Thermoelectric Efficiency: A Dynamical Systems Approach
}

\author{
Giulio Casati, ${ }^{1,2}$ Carlos Mejía-Monasterio, ${ }^{3}$ and Tomaž Prosen ${ }^{4}$ \\ ${ }^{1}$ Center for Nonlinear and Complex Systems, Università degli Studi dell'Insubria, Como, Italy \\ ${ }^{2}$ CNR-INFM and Istituto Nazionale di Fisica Nucleare, Sezione di Milano, Milan, Italy \\ ${ }^{3}$ Département de Physique Théorique, Université de Genève, Geneva, Switzerland \\ ${ }^{4}$ Physics Department, Faculty of Mathematics and Physics, University of Ljubljana, Ljubljana, Slovenia
}

(Received 27 February 2008; published 2 July 2008)

\begin{abstract}
Inspired by the kinetic theory of ergodic gases and chaotic billiards, we propose a simple microscopic mechanism for the increase of thermoelectric efficiency. We consider the cross transport of particles and energy in open classical ergodic billiards. We show that, in the linear response regime, where we find exact expressions for all transport coefficients, the thermoelectric efficiency of ideal ergodic gases can approach the Carnot efficiency for sufficiently complex charge carrier molecules. Our results are clearly demonstrated with a simple numerical simulation of a Lorentz gas of particles with internal rotational degrees of freedom.
\end{abstract}

DOI: 10.1103/PhysRevLett.101.016601

PACS numbers: 72.15.Jf, 05.45.-a, 05.70.Ln

Although thermoelectricity was discovered almost 200 years ago, a strong interest of the scientific community arose only in the 1950s when Abram Ioffe discovered that doped semiconductors exhibit a relatively large thermoelectric effect. This initiated intense research activity in semiconductor physics which was not motivated by microelectronics but by the Ioffe suggestion that home refrigerators could be built with semiconductors [1,2]. As a result of these efforts, the thermoelectric material $\mathrm{Bi}_{2} \mathrm{Te}_{3}$ was developed for commercial purposes. However, this activity lasted only a few years until the mid 1960s since, in spite of all efforts and consideration of all types of semiconductors, it turned out that thermoelectric refrigerators still have poor efficiency as compared to compressor-based refrigerators. Because of environmental concerns, in the past decade there has been increasing pressure to find better thermoelectric materials with higher efficiency.

The suitability of a thermoelectric material for energy conversion or electronic refrigeration is evaluated by the thermoelectric figure of merit $Z$ :

$$
Z=\frac{\sigma S^{2}}{\kappa},
$$

where $\sigma$ is the coefficient of electric conductivity, $S$ is the Seebeck coefficient, and $\kappa$ is the thermal conductivity. The Seebeck coefficient $S$, also called thermopower, is a measure of the magnitude of an induced thermoelectric voltage in response to a temperature difference across the material.

For a given material, and a pair of temperatures $T_{H}$ and $T_{C}$ of hot and cold thermal baths, respectively, $Z$ is related to the efficiency $\eta$ of converting the heat current $J_{Q}$ (between the baths) into the electric power $P$ which is generated by attaching a thermoelectric element to an optimal Ohmic impedance. Namely, in the linear regime:

$$
\eta=\frac{P}{J_{Q}}=\eta_{\text {carnot }} \frac{\sqrt{Z T+1}-1}{\sqrt{Z T+1}+1},
$$

where $\eta_{\text {carnot }}=1-T_{C} / T_{H}$ is the Carnot efficiency and
$T=\left(T_{H}+T_{C}\right) / 2$. Thus a good thermoelectric device is characterized by a large value of the nondimensional figure of merit $Z T$.

Since the 1960s many materials have been investigated, but the maximum value found for $Z T$ was achieved for the $\left(\mathrm{Bi}_{1-x} \mathrm{Sb}_{x}\right)_{2}\left(\mathrm{Se}_{1-y} \mathrm{Te}_{y}\right)_{3}$ alloy family with $Z T \approx 1$. However, values $Z T>3$ are considered to be essential for thermoelectrics to compete in efficiency with mechanical power generation and refrigeration at room temperatures. The efforts recently focused on a bulk of new advanced thermoelectric materials and on low-dimensional materials, and only a small increment of the efficiency $Z T \lesssim 2.6$ has been obtained [3].

One of the main reasons for this partial success is a limited understanding of the possible microscopic mechanisms leading to the increase of $Z T$, with few exceptions [4]. From a dynamical point of view, cross effects in transport have been barely studied [5,6]. So far, the challenge lies in engineering a material for which the values of $S, \sigma$, and $\kappa$ can be controlled independently. However, the different transport coefficients are interdependent, making optimization extremely difficult.

By considering a Lorentz gas system in this Letter, we show that large values of $Z T$, in principle approaching Carnot's efficiency, can be obtained when the energy of the carrier particles does not depend on the thermodynamic forces.

In the linear response regime (see, e.g., [7]), one writes the heat current $J_{Q}$ and the electric current $J_{e}$ through a homogeneous sample subjected to a temperature gradient $\partial_{x} T$ and an electrochemical potential gradient $\partial_{x} \bar{\mu}$ as

$$
\begin{aligned}
J_{Q} & =-\kappa^{\prime} \partial_{x} T-T \sigma S \partial_{x} \bar{\mu}, \\
J_{e} & =-\sigma S \partial_{x} T-\sigma \partial_{x} \bar{\mu} .
\end{aligned}
$$

Here and in what follows, we assume that the transport occurs along the $x$ direction and the temperature is given in units where the Boltzmann constant $k_{B}=1$. 
The electrochemical potential is the sum of a chemical and an electric part $\bar{\mu}=\mu+\mu_{e}$, where $\mu$ is the chemical potential of the particles and, if $e$ is the particle's charge, $\mu_{e}=e \phi$ is the work done by the particles against an external electric field $\mathcal{E}=-\partial_{x} \phi$. From (3) the usual phenomenological relations follow: If the thermal gradient vanishes, $\partial_{x} T=0$, then $J_{e}=-\sigma \partial_{x} \phi=\sigma \mathcal{E}$, since for an isothermal homogeneous system $\mu$ is uniform. If the electric current vanishes, $J_{e}=0$, then $\partial_{x} \bar{\mu}=S \partial_{x} T$, which is the definition of the Seebeck coefficient, and $J_{Q}=$ $-\kappa \partial_{x} T$, where $\kappa=\kappa^{\prime}-T \sigma S^{2}$ is the usual thermal conductivity (see, e.g., [8]). From the theory of irreversible thermodynamics, $\mu$ and $\mu_{e}$ cannot be determined separately; only their combination $\bar{\mu}$ appears in (3) [9]. Based on this equivalence, in what follows we take into account the chemical part only, i.e., $\bar{\mu}=\mu$.

Our aim is to study thermoelectricity from an "energy transport" point of view. To linear order, the energy and particle density currents $J_{u}$ and $J_{\varrho}$, respectively, can be written in terms of the Onsager matrix $\mathbb{L}[8,10]$ as

$$
\begin{aligned}
& J_{u}=L_{u u} \partial_{x}\left(\frac{1}{T}\right)+L_{u \varrho} \partial_{x}\left(-\frac{\mu}{T}\right), \\
& J_{\varrho}=L_{\varrho u} \partial_{x}\left(\frac{1}{T}\right)+L_{\varrho \varrho} \partial_{x}\left(-\frac{\mu}{T}\right),
\end{aligned}
$$

where $J_{e}=e J_{\varrho}$. In the absence of magnetic fields, the Onsager reciprocity relations states that $\mathbb{L}$ is symmetric, $L_{u \varrho}=L_{\varrho u}$. From the entropy balance equation for open systems $J_{u}=J_{Q}+\mu J_{Q}$. Substituting $J_{Q}$ in (3) in favor of $J_{u}$ and comparing the resulting equations with (4), it follows that the transport coefficients can be written in terms of the $L$ coefficients as

$$
\sigma=\frac{e^{2}}{T} L_{\varrho \varrho}, \quad \kappa=\frac{1}{T^{2}} \frac{\operatorname{det} \mathbb{L}}{L_{\varrho \varrho}}, \quad S=\frac{1}{e T}\left(\frac{L_{u \varrho}}{L_{\varrho \varrho}}-\mu\right) .
$$

Equations (4) and (5) are completely equivalent to the description (3), and, using Eq. (1), we obtain for the figure of merit

$$
Z T=\frac{\left(L_{u \varrho}-\mu L_{\varrho \varrho}\right)^{2}}{\operatorname{det} \llbracket} .
$$

Note that, in Eqs. (5) and (6), $T$ and $\mu$ are taken as the mean values. Expressions (4) and (6) provide a very convenient way for numerical or analytical evaluation of $Z T$ for different kinds of dynamical models.

From (6) it is clear that the second law does not impose any upper bound on the value of $Z T$. It requires only that $\mathbb{L}$ is positive definite. Furthermore, the crucial observation is that Carnot's limit $Z T=\infty$ is reached when the energy density current and the electric current become proportional, since then det $\mathbb{L}=0$. Suppose, for example, that both energy and charge are carried only by noninteracting particles, like in a dilute gas. Then the microscopic instantaneous currents per particle at position $x^{*}$ and time $t$ are

$$
\begin{aligned}
& j_{u}\left(x^{*}, t\right)=E(t) v_{x}(x(t), t) \delta\left(x^{*}-x(t)\right), \\
& j_{e}\left(x^{*}, t\right)=e v_{x} \delta\left(x^{*}-x(t)\right),
\end{aligned}
$$

where $E$ is the energy of the particle, $x$ its position, and $v_{x}$ its velocity along the field. The thermodynamic averages of the two currents [appearing in Eq. (4)] become proportional when the variables $E$ and $v_{x}$ are uncorrelated

$$
J_{u}=\left\langle j_{u}\right\rangle=\langle E\rangle\left\langle v_{x}\right\rangle=\frac{\langle E\rangle}{e}\left\langle j_{e}\right\rangle=\langle E\rangle J_{\varrho} .
$$

Therefore, $Z T=\infty$ follows from the fact that the average particle's energy $\langle E\rangle$ does not depend on the thermodynamic forces. In the context of classical physics, this happens, for instance, in the limit of large number of internal degrees of freedom (DOF), provided the dynamics is ergodic.

We consider an ergodic gas of noninteracting, electrically neutral particles of mass $m$ with $d_{\text {int }}$ internal DOF (rotational or vibrational), in a $d$-dimensional container [11]. To study the nonequilibrium state of such dilute polyatomic gas, we consider a chaotic billiard (Fig. 1) connected through openings of length $\lambda$ to two reservoirs of particles, idealized as infinite chambers with the same polyatomic gas at equilibrium density $\varrho$ and temperature $T$. From the reservoirs, particles are injected into the channel at a rate $\gamma$ obtained by integration over energy of the appropriate canonical distribution to give

$$
\gamma=\frac{\lambda}{(2 \pi m)^{1 / 2}} \varrho T^{1 / 2} .
$$

The particle injection rate $\gamma$ is related to the value of the chemical potential $\mu$ at the reservoirs which, for a gas of polyatomic molecules with a total of $D=d+d_{\text {int }}$ DOF, at equilibrium density $\varrho$ and temperature $T$ reads

$$
\mu=T \ln \left(\frac{c_{D}^{\prime} \varrho}{T^{D / 2}}\right)=T \ln \left(\frac{c_{D} \gamma}{T^{(D+1) / 2}}\right),
$$

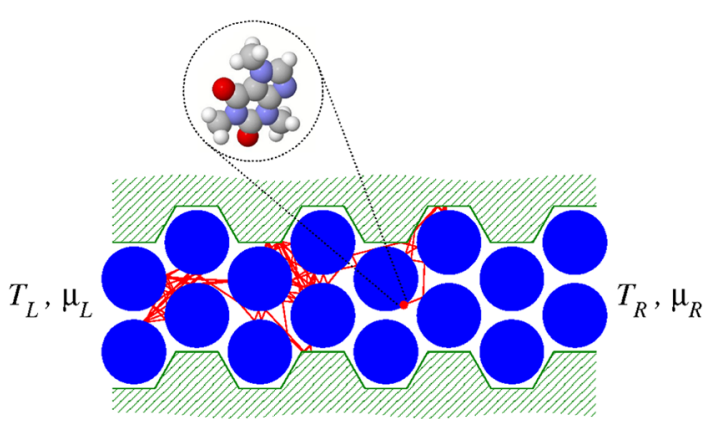

FIG. 1 (color online). The open Lorentz gas system and a typical particle's trajectory. The composite particle (schematically represented as a molecule) is scattered from fixed disks of radius $R$ disposed in a triangular lattice at the critical horizon; i.e., the width and height of the cells are $\Delta x=2 R$ and $\Delta y=$ $2 W$, respectively, where $W=4 R / \sqrt{3}$ is the separation between the centers of the disks. The channel is coupled at the left and right boundaries to two thermochemical baths at temperatures $T_{L}$ and $T_{R}$ and chemical potentials $\mu_{L}$ and $\mu_{R}$, respectively. 
where $c_{D}^{\prime}$ and $c_{D}$ are $D$-depending constants and the second equality is obtained by substituting $\varrho$ from Eq. (9). Furthermore, by averaging the energy of the injected particles over the canonical distribution, denoted as $\langle E\rangle$, we obtain the rate at which energy is injected from the reservoirs as $\varepsilon=\gamma\langle E\rangle=\gamma T(D+1) / 2$. Let $p_{t}(l)$ be the transmission probability of the channel of length $l$. For a billiard system of noninteracting particles [12], the density currents $J_{u}, J_{\varrho}$ assume a simple form: They are $p_{t}(l)$ times the difference between the left and right corresponding injection rates $\varepsilon$ and $\gamma$, respectively, namely,

$$
J_{\varrho}=p_{t}\left(\gamma_{L}-\gamma_{R}\right), \quad J_{u}=p_{t}\left(\varepsilon_{L}-\varepsilon_{R}\right) .
$$

Using (10) to eliminate $\gamma$ in favor of $\mu$, we obtain

$$
\begin{aligned}
J_{\varrho} & =-\frac{\lambda p_{t}(l) l}{(2 \pi m)^{1 / 2}} \partial_{x}\left(T^{(D+1) / 2} e^{\mu / T}\right), \\
J_{u} & =-\frac{\lambda p_{t}(l) l}{(2 \pi m)^{1 / 2}} \frac{D+1}{2} \partial_{x}\left(T^{(D+3) / 2} e^{\mu / T}\right) .
\end{aligned}
$$

By taking total differentials of (12) in $1 / T$ and $\mu / T$ and comparing with Eq. (4), we obtain exact microscopic expressions for the Onsager coefficients, namely,

$$
\begin{aligned}
& L_{\varrho \varrho}=\frac{\lambda p_{t}(l) l}{(2 \pi m)^{1 / 2}} \varrho T^{1 / 2} \\
& L_{\varrho u}=L_{u \varrho}=\frac{\lambda p_{t}(l) l}{(2 \pi m)^{1 / 2}}\left(\frac{D+1}{2}\right) \varrho T^{3 / 2} \\
& L_{u u}=\frac{\lambda p_{t}(l) l}{(2 \pi m)^{1 / 2}} \frac{(D+1)(D+3)}{4} \varrho T^{5 / 2} .
\end{aligned}
$$

Note that, for a chaotic billiard channel with a diffusive dynamics, the transmission probability decays as $p_{t}(l) \propto$ $l^{-1}$ which means that all of the elements of the Onsager matrix $\mathbb{L}$ become size-independent. Finally, by plugging (13) into (6) and noting that $c_{V}^{*}=D / 2$ is the dimensionless heat capacity at a constant volume of the gas, we obtain

$$
Z T=\frac{1}{\hat{c}_{V}}\left(\hat{c}_{V}-\frac{\mu}{T}\right)^{2},
$$

where for simplicity we have set $\hat{c}_{V}=c_{V}^{*}+1 / 2$. A particular case of (14) was previously obtained, for noninteracting monoatomic ideal gases in 3 dimensions [13]. In the absence of particles interaction, $Z T$ is independent of the sample size $l$ and depends on the temperature only through the chemical potential term. This is due to the fact that, with no interactions, $p_{t}$ depends on the geometry of the billiard only. From a physical point of view, this means that the mean free path of the gas particles is energyindependent. Were particles interacting, $p_{t}$ would depend on the local density and temperature of the gas, leading to a more realistic situation [14]. Interestingly enough, we have found that Eq. (14) is an upper limit of the interacting case when the interaction strength vanishes [15]. Indeed, the interaction tends to increase the deviation from the uncorrelated situation (8), and therefore it is to be expected that, considering only transporting particles' classical degrees of freedom, a particle-particle interaction decreases the thermoelectric efficiency.

We shall now confirm Eq. (14) with a very simple numerical demonstration of a two-dimensional chaotic Lorentz gas channel of particles elastically colliding with circular obstacles of radius $R$. In what follows, we fix the unit length setting $R=1$. The geometry of the model is shown in Fig. 1. We consider composite particles with $d_{\text {int }} \geq 1$ internal rotational DOF. Each "particle" of mass $m$ can be imagined as a stack of $d_{\text {int }}$ small identical disks of mass $m / d_{\text {int }}$ and radius $r \ll R$, rotating freely and independently at a constant angular velocity $\omega_{i}, i=1, \ldots, d_{\text {int }}$. The center of mass of the particle moves with velocity $\vec{v}=$ $\left(v_{x}, v_{y}\right)$. At each collision of the particle with the boundary of the billiard (either one of the circular obstacles or the outer wall), an energy exchange among all of the $D$ DOF occurs according to the following collision rules: $v_{n}^{\prime}=$ $-v_{n}$ and

$$
\begin{aligned}
v_{t}^{\prime} & =\frac{1-\eta d_{\mathrm{int}}}{1+\eta d_{\mathrm{int}}} v_{t}+\frac{2 \eta}{1+\eta d_{\mathrm{int}}} \sum_{k=1}^{d_{\mathrm{int}}} \omega_{k}, \\
\omega_{i}^{\prime} & =\frac{2}{1+\eta d_{\mathrm{int}}} v_{t} \sum_{k=1}^{d_{\mathrm{int}}}\left(\delta_{i k}-\frac{2}{d_{\mathrm{int}}\left(1+\eta d_{\mathrm{int}}\right)}\right) \omega_{k},
\end{aligned}
$$

where $\left(v_{n}, v_{t}\right)$ are the normal and tangent components, respectively, of the particle velocity at the collision point and $\eta=\Theta / m r^{2}$, with $\Theta$ being the moment of inertia of each small fictitious disk. The primed (unprimed) quantities refer to the values after (before) the collision. These collision rules are a generalization of the ones introduced in Ref. [6]. They are deterministic and time reversible and preserve the energy and total angular momentum [15].

First we considered a closed system and checked energy equipartition among all DOF. Then we have opened the system from both ends and allowed it to exchange particles with the two baths at temperatures $T_{L}$ and $T_{R}$ and with chemical potentials $\mu_{L}$ and $\mu_{R}$. The coupling among the system and the baths is defined as follows: Whenever a particle in the system crosses the opening which separates it from the bath, it is removed from the system. On the other hand, with frequency $\gamma$, particles are injected into the system, with an energy distributed according to the canonical distribution at the corresponding temperature. By two different simulations with two linearly independent sets of thermodynamic forces, we have numerically determined the $\mathbb{L}$ matrix and the value of $Z T$ (see Fig. 2). Numerical results excellently confirm the theoretical prediction (14). We have also carefully checked that all Onsager coefficients, or conductivities, decay with the size $l$ of the system as $1 / l$ which indicates a diffusive transport.

The simple mechanism for the growth of $Z T$ with $d_{\text {int }}$ is nicely illustrated in Fig. 3, which shows that the particle velocity $v_{x}$ has a Maxwellian (Gaussian) distribution (inset), while the equilibrium distribution of the particle energy per degree of freedom $E_{D}$ becomes more and 


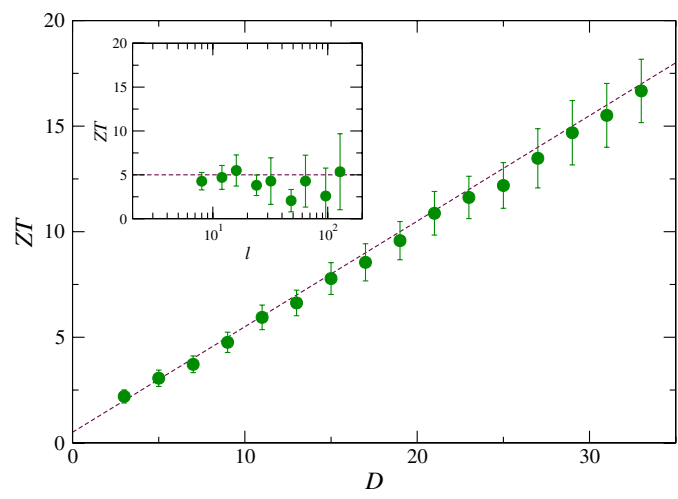

FIG. 2 (color online). Figure of merit $Z T$ as a function of the number of DOF, at $\mu=0$. For each $D$, the transport coefficients were obtained from two different simulations in a channel of 10 cells, at mean particle density $\varrho=0.446$ and mean temperature $T=1000$ with (a) $\Delta T / T=0.04, \Delta(\mu / T)=0$ and (b) $\Delta T=0, \Delta(\mu / T)=0.04$. The injection rates $\gamma_{L}$ and $\gamma_{R}$ are obtained from $\Delta(\mu / T)$, using (9) and (10). The dashed line corresponds to $(D+1) / 2$. In the inset, the dependence of $Z T$ on the length of the channel $l$ is shown for $D=9$. The dashed line shows the corresponding expected value.

more sharply peaked and thus decorrelated from $v_{x}$ as $d_{\text {int }}$ grows. This property allows a qualitative understanding of the behavior of transport coefficients. Indeed, by substituting Eqs. (13) in Eqs. (5), one obtains that, while the electric conductivity $\sigma$ does not scale with $D$, both the heat conductivity $\kappa$ and the Seebeck coefficient scale linearly in $D$. Therefore, for a given temperature gradient, the heat current increases linearly in $D$, while the electric current

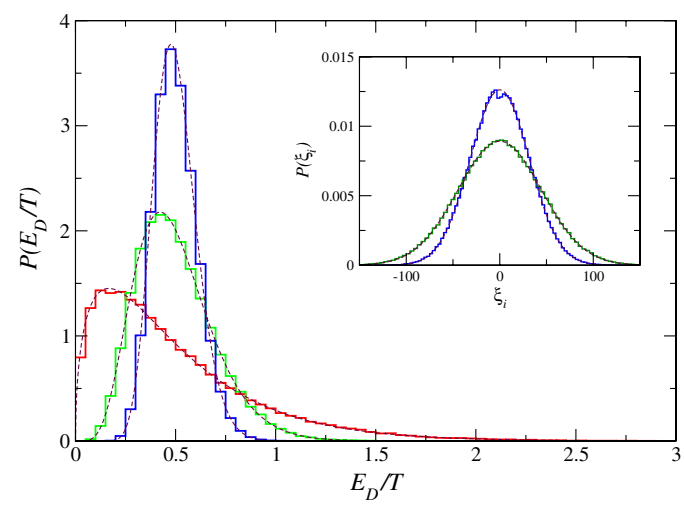

FIG. 3 (color online). Probability distribution function of the energy per degree of freedom $E_{D}=E / D$ determined from equilibrium simulation with $T_{L}=T_{R}$ and $\mu_{L}=\mu_{R}$, for different numbers of freedoms: $D=3$ (red curve), $D=13$ (green curve), and $D=43$ (blue curve). The dashed curves are the theoretical ("Chi-square" $\chi_{D}^{2}$ ) distributions of $E_{D}$. In the inset, the corresponding probability distribution functions for the $x$ component of the velocity $P\left(v_{x}\right)$ (blue curve) and for the angular momentum of one of the particle's disks $P\left(\omega_{i}\right)$ (green curve) is shown, for $D=3$. The dashed curves are the theoretical Gaussian distributions. remains unaffected. However, since $S$ does also scale as $\sim D$, the efficiency increases [16].

In conclusion, we have discovered a simple general theoretical mechanism which may find a way to applications of thermoelectricity in real world materials. Even though the case of an ionized polyatomic gas may seem a little artificial in this context, there may be other important instances where each charge would be carried by many effectively classical DOF. We have also performed the first numerical computation of $Z T$ from deterministic microscopic equations of motion. Our method can easily be implemented for more realistic models where quantum effects can also be taken into account.

The authors are indebted to $\mathrm{H}$. Linke and C. Vining for enlightening discussions and correspondence, and thank the hospitality of the Institut Henri Poincaré, Paris, where part of this work was done. T.P. acknowledges support from Grants No. P1-0044 and No. J1-7347 of the Slovenian research agency. C.M.M. acknowledges support from Fonds National Suisse and from Fondazione CRT.

[1] G. Mahan, B. Sales, and J. Sharp, Phys. Today 50, No. 3, 42 (1997).

[2] A. Majumdar, Science 303, 777 (2004).

[3] M.S. Dresselhaus et al., Adv. Mater. 19, 1043 (2007).

[4] T. E. Humphrey and H. Linke, Phys. Rev. Lett. 94, 096601 (2005).

[5] C. Maes and M.H. van Wieren, J. Phys. A 38, 1005 (2005); J. Vollmer, T. Tell, and L. Mátyás, J. Stat. Phys. 101, 79 (2000); C. Van den Broeck, in Advances in Chemical Physics, edited by S. A. Rice (Wiley, New York, 2007), Vol. 135.

[6] C. Mejia-Monasterio, H. Larralde, and F. Leyvraz, Phys. Rev. Lett. 86, 5417 (2001).

[7] D. J. Bergman and O. Levy, Appl. Phys. 70, 6821 (1991).

[8] C. A. Domenicali, Rev. Mod. Phys. 26, 237 (1954).

[9] P. L. Walstrom, Am. J. Phys. 56, 890 (1988); see also H. Larralde, F. Leyvraz, and C. Mejia-Monasterio, J. Stat. Phys. 113, 197 (2003), where the equivalence between $\mu$ and $\mu_{e}$ has been studied in a similar model.

[10] H. B. Callen, Phys. Rev. 73, 1349 (1948); S. R. de Groot and P. Mazur, Non-equilibrium Thermodynamics (Dover, New York, 1984).

[11] If energy, mass, and electric charge are carried by particles, thermodiffusion and thermoelectricity are related phenomena. Moreover, in the linear regime in which dissipative effects, such as, e.g., Joule heating, can be neglected and at low particle densities, thermodiffusion and thermoelectricity are equivalent processes.

[12] Assuming that particles do not interact means that we fully neglect the phonon transport.

[13] C. B. Vining, Mater. Res. Soc. Symp. Proc. 478, 3 (1997).

[14] J.-P. Eckmann, C. Mejia-Monasterio, and E. Zabey, J. Stat. Phys. 123, 1339 (2006).

[15] G. Casati, C. Mejia-Monasterio, and T. Prosen (to be published).

[16] We remark that the increase of $Z$ is not related with the validity of the fluctuation theorems. 